\title{
Träume von Kindern in Diagnose und Psychotherapie
}

\author{
Hans Hopf (Mundelsheim)
}

Zusammenfassung: Träume von Kindern finden in der Kinderpsychoanalyse nicht jene Beachtung, wie das bei Erwachsenen geschieht. Eine Ursache dafür ist, dass kleinere Kinder seltener Träume erzählen, vor allem jedoch, dass sie kaum Assoziationen dazu äussern. Freud verstand die Träume von kleinen Kindern als einfache, meist an ein Vortagsereignis anknüpfende unverhüllte Wunscherfüllungen. Er erwähnte auch, dass bis zum fünften Lebensjahr noch manifester und latenter Trauminhalt zusammenfielen. Tatsächlich hängt die Strukturierung eines Traumes von der kognitiven Entwicklung ab: Die Möglichkeiten von Kindern, sich auszudrücken, sind noch begrenzt; was sie ausdrücken wollen, unterscheidet sich von dem, was sie ausdrücken können. Doch betrifft das nicht die Funktionen, die Träume übernehmen können. Diese sind neben der Wunscherfüllung ähnliche wie bei Jugendlichen und Erwachsenen, etwa die Funktion der Selbstdarstellung und die kommunikative Funktion, in denen eher dynamische Aspekte deutlich werden. An zwei Beispielen wird aufgezeigt, wie es möglich ist, auch mit Träumen von Kindern psychoanalytisch zu arbeiten. Eines der Beispiele ist ein Traum nach traumatischem Erleben; diese traumatischen Träume weisen eine ganz besondere Struktur auf. Einbezogen in den Artikel ist eine Diskussion der Gedanken von Fritz Morgenthaler zur Theorie und Technik, die für den Umgang mit Träumen von Kindern von höchster Bedeutung sind.

Schlüsselwörter: Kindertraum, Diagnostik, Deutung, Kinderpsychoanalyse

\section{Der Kindertraum und Freuds Traumdeutung}

Die Geschichte des Kindertraums beginnt bekanntlich bei Sigmund Freud mit einer Reihe von Träumen, zum Teil von seinen eigenen Kindern, die infantile Wunscherfüllungen enthalten. Das jüngste Kind unter den kleinen Träumerinnen und Träumern war die neunzehnmonatige Anna. Sie hatte im Jahr 1896 eines Nachts energisch jene Nahrung eingefordert, die ihr tagsüber von der Kinderfrau verweigert worden war, weil sie morgens erbrochen hatte: «Anna Feud, Er(d) beer, Hochbeer, Eier(s)peis, Papp» (Freud, 1900a, S. 148), vielleicht zu verstehen 
als: «Ich will essen, was ich will und niemand soll mich daran hindern.» Es ist der Traum eines Kindes während seiner Autonomieentwicklung. Freud betrachtete diese Sorte von Träumen allerdings ausschliesslich als simple, unverkleidete Wunscherfüllungen, die er im Gegensatz zu den Träumen Erwachsener gar nicht interessant fand (vgl. 1900a, S. 145).

Sigmund Freud (1916/1917) charakterisierte die Träume der Kinder bis etwa zum fünften Lebensjahr als kurz, klar, kohärent, leicht zu verstehen und unzweideutig. Er verstand die Kinderträume als einfache, meist an ein Vortagesereignis anknüpfende unverhüllte Wunscherfüllungen und erwähnte - wie oben gesagt -, dass bis zum fünften Lebensjahr manifester und latenter Trauminhalt zusammenfielen, erst dann setze in der Regel die Traumentstellung ein und die Träume würden komplizierter. Darum waren die Träume der kleinen Kinder für Freud auch nicht interessant, ausser dass sie für das Studium der Träume von Erwachsenen von Nutzen sein könnten, was er mit den folgenden Worten bekräftigte:

Die allereinfachsten Formen von Träumen darf man wohl bei Kindern erwarten, deren psychische Leistungen sicherlich minder kompliziert sind als die Erwachsener. Die Kinderpsychologie ist nach meiner Meinung dazu berufen, für die Psychologie der Erwachsenen ähnliche Dinge zu leisten wie die Untersuchungen des Baues oder der Entwicklung niederer Tiere für die Erforschung der Struktur der höchsten Tierklassen. Es sind bis jetzt wenig zielbewusste Schritte geschehen, die Psychologie der Kinder zu solchem Zwecke auszunützen. (Freud, 1900a, S. 145)

Dieses Zitat verdeutlicht Freuds ernsthafte Bemühungen, über den Kindertraum zu einem tieferen Verständnis des Traumes zu gelangen. Die herangezogenen Bilder - etwa der Vergleich von Kindern mit niederen Tieren - verraten jedoch auch ein zum damaligen Zeitpunkt ambivalentes Verhältnis zum Kind und seinen Besonderheiten.

\section{Vom Verschwinden des Kindertraums}

Der Kindertraum konnte auch später nie jene zentrale Bedeutung für die Kinderanalyse gewinnen, wie sie die Träume der Erwachsenen in der Psychoanalyse einnahmen. Nach Auffassungen von Despert (1949) sowie Ablon und Mack (1980) rührte das vor allem daher, dass Kinder ungern Träume berichten würden. Diese Feststellung ist jedoch nicht richtig. Haben sich eine Bindung sowie ein stabiles
Behandlungsbündnis etabliert, erzählen Kinder den Therapeutinnen und Therapeuten gerne ihre Träume.

Eine weitere Ursache dafür, dass Träume in der Kinderpsychoanalyse kaum angewandt würden, sahen manche Autoren darin, dass Kinder in der Wiedergabe ihrer Träume ungenau und unzuverlässig seien. Beispielsweise schrieb hierzu Zierl (1973, S. 415): «Erinnerungsfälschungen, Umdichtungen nach Massgabe des Wachbewusstseins, konfabulatorische Ausschmückungen und Elemente des Wunschdenkens können den Traumtext bis zur Unkenntlichkeit modifizieren.»

Tatsächlich sind die Übergänge zwischen Traumbericht, Tagtraum und Fantasien fliessend und daher ist es bei Kinderträumen oft nicht möglich, den Unterschied zwischen eigentlichem Traumbericht und im Nachhinein produzierten Fantasien und Ausschmückungen auszumachen. Innerhalb der Kinderpsychoanalyse wiegt diese Tatsache letztlich gering; denn nach psychoanalytischem Verständnis gelten auch Fantasien und Tagträume als mehr oder weniger verkleidete Wunscherfüllungen, als Ersatz für Versagungen in der Realität und besitzen darum die gleiche Funktion wie nächtliches Träumen - nämlich jene, psychische Spannungen abzureagieren (vgl. Freud, A., 1978, S. 2834).

Ein entscheidender Grund für die stiefmütterliche Behandlung des Traumes in der Psychoanalyse des Kindes resultiert aus einer anderen Entdeckung: Da sich nach psychoanalytischer Auffassung Triebabkömmlinge, Impulse und Wünsche auch im freien Spiel, in bewussten Fantasien und in Tagträumen ausleben, erübrige sich in den meisten Fällen eine konsequente Traumanalyse.

Es war darum weniger die Weigerung von Kindern, Träume zu berichten, auch nicht ihre Neigung zur konfabulatorischen Ausschmückung: Es war der Ausfall der freien Assoziation, der die Psychoanalyse auf eine konsequente Nutzung des Kindertraumes verzichten liess: Kinder verweigern die analytische Grundregel, ihre Träume regelmässig und kritiklos mitzuteilen. Wenn sie gelegentlich Träume in die psychoanalytische Behandlung bringen, liefern sie im Gegensatz zu Erwachsenen selten Einfälle zu den einzelnen Traumelementen. Anna Freud (1965) beschrieb diesen Sachverhalt in folgender Weise: «Sie teilen ihre Erlebnisse mit dem Analytiker, vorausgesetzt, dass ein Vertrauensverhältnis innerhalb der Analyse hergestellt ist; aber ohne das Mittel der freien Assoziation können ihre Mitteilungen nicht über den Rahmen des Bewusstseins hinausgehen» (1965, S. 2149 f.).

Anna Freud führt diesen Ausfall des freien Assoziierens zum einen darauf zurück, dass die Position des Erwachsenen als Autoritäts- und Über-Ich-Figur eine uneingeschränkte Aufrichtigkeit des Kindes verhindere. Zum anderen misstraue das kindliche und unreife Ich der eigenen Widerstandskraft dem Triebleben 
gegenüber: Eine Ausschaltung von Kritik und Zensur bedeutet somit eine grössere Gefahr für das Kind als für den Erwachsenen. So wurden Träume innerhalb der Kinderpsychoanalyse immer weniger einbezogen; seit Hermine Hug-Hellmuth, Anna Freud und Melanie Klein wurde das kindliche Spiel als der Ausdruck unbewusster Konflikte angesehen und trat zunehmend in den Mittelpunkt, umgekehrt wurde eine konsequente Analyse von Kinderträumen immer seltener durchgeführt (Burchartz, Hopf et al., 2016, S. 34 f.).

\section{Psychoanalytische Arbeit auch mit Träumen von Kindern?}

Ich kehre nochmals zu Freuds Feststellung zurück, dass Kinderträume einfache Wunscherfüllungen seien, dass manifester und latenter Trauminhal zusammenfielen und sie darum nicht interessant seien. Mit dieser Feststellung hat Freud teilweise Recht, denn Kindern stehen die Mechanismen der Traumarbeit und die Fähigkeiten für die Umwandlungsarbeit der Traumzensur (Verdichtung, Verschiebung, Verkehrung ins Gegenteil und vor allem die Symbolisierung) noch nicht ausreichend zur Verfügung, ihre Träume sind deshalb «nieder strukturiert». Ursache dafür ist die noch nicht abgeschlossene kognitive Entwicklung von kleinen Kindern. Erst ab dem sechsten Lebensjahr wird Kindern klar, dass Träume in ihrem Inneren stattfinden und eine Art Gedanken darstellen, erst danach wird der Traum als innerseelisches Geschehen verstanden. Natürlich hängt die Länge der Traumerzählungen auch mit dem zunehmenden Wortschatz und der Grammatisierung der Sprache zusammen, denn die Gestaltung des manifesten Traums (oder eines Narrativs) hängt von den sprachlichen Möglichkeiten ab. Die Möglichkeiten von Kindern, sich auszudrücken, sind noch begrenzt; was sie ausdrücken wollen, unterscheidet sich von dem, was sie ausdrücken können. Auch weist die Traumerzählung eines Kindes in der Regel Lücken auf, weil solche Traumteile als absurd verworfen werden, die konträr zu den bisherigen eigenen Erfahrungen stehen.

Aber so eindeutig ist das alles nicht. Kinder können in ihren Träumen sehr wohl symbolisieren, sie gehen dabei aber anders vor. In fast drei Viertel der Träume von kleinen Kindern tummeln sich Tiere aller Arten. Ihre Anzahl lässt mit zunehmendem Alter nach, bei Erwachsenen sind es gerade noch 7.5 Prozent. Tiere eignen sich bestens als Projektionsflächen für Affekte und komplexe Vorstellungen. Darum besitzen sowohl der Bär, der Wolf als auch die Schlange im Märchen symbolischen Charakter. Vor allem die Schlange zeigt vielfältige symbolische Facetten und Bedeutungen: Im primitiven Bewusstsein sind Tier und Mensch innerlich noch austauschbar
Es ist davon auszugehen, dass die Abnahme von Tieren in Träumen auf eine immer reifere Symbolisierungsfähigkeit der heranwachsenden Träumerinnen und Träumer zurückzuführen ist. Ein kleines Kind ist anfänglich nur bedingt fähig, sich und seine Affekte darzustellen. Es projiziert darum seine affektiven Vorstellungen auf das Tier, so dass es zu einer noch symbolisch gleichsetzenden Darstellung von Affekten kommt (etwa Krokodil=Aggression; Hase=Angst; Schäfchen= Bedürfnis nach Nähe). Damit können Tiere in Träumen im Verlauf der Entwicklung auch die Funktion von Übergangsobjekten, hin zur reifen Symbolisierung und Objektbeziehung einnehmen: Wird ein Kind älter, so stehen ihm immer reifere Symbole zur sublimen Abbildung seiner Gefühlswelt zurVerfügung (vgl. Hopf, 2007, S. 77 f.)

\section{Ein erstes Beispiel für einen Kindertraum}

Seit ich kinderpsychoanalytisch arbeite, ist es mir ein Anliegen, Träume von Kindern wieder in Behandlungen einzubeziehen. Bei genauerer Betrachtung gibt es keine überzeugenden Gründe, Kinderträume nicht zu nutzen.

Wenn Träume von Kindern lediglich unter dem Aspekt derWunscherfüllung betrachtet werden, wird das wenig ergiebig sein. Erikson hat bereits 1955 die einseitige Fixierung auf den latenten Trauminhalt kritisiert, indem er schrieb: «Offiziell aber sind wir bei jedem Traum, vor den wir gestellt sind, sehr schnell damit bei der Hand, seine manifeste Gestalt aufzuknacken wie eine nutzlose Nussschale, die wir eilends wegwerfen, um zu dem scheinbar so wertvolleren Kern zu gelangen» (1955, S. 31). Mit dem Wort «offiziell» hat Erikson angedeutet, dass man sich mit einem solchen Vorgehen dem Freud'schen Dogma von der alleinigen Bedeutung des latenten Trauminhaltes gebeugt hatte. Zwar akzeptierte auch er, dass der latente infantile Wunsch die Energie für den wiedererwachten Konflikt und damit den Traum liefern würde, allerdings in eine manifeste Traumstruktur eingebettet, die auf jeder Ebene bezeichnende Züge der Gesamtsituation des Träumers widerspiegeln würde (1955, S. 72). Vor dem Hintergrund der Entwicklung der psychoanalytischen Theorie von einer Psychologie des Unbewussten zu einer Ich-Psychologie und schliesslich zu einer Objektbeziehungspsychologie gewann auch der manifeste Kindertraum für Diagnose und Therapie an Bedeutung, weil der Ausfall der freien Assoziation nicht mehr entscheidend ins Gewicht fiel (vgl. Hopf, 1985).

Entscheidend war jedoch, dass mittlerweile vielerlei Funktionen und Zugangswege zum Traum beschrieben worden waren, die ich im Verlauf dieses Artikels noch herausarbeiten werde. Sehr beeindruckt haben mich dabei die Überlegungen von Fritz Morgenthaler, denen ich in den neunziger Jahren begegnet bin. In seinem Artikel «Traumdiagnostik» (Morgenthaler, 1990) berichtet er über 
einen Kindertraum, den ihm eine Patientin von ihrem Kind berichtet hatte. Es hat seinen Eltern beim Kirschenpflücken geholfen. Weil es brav und wohlerzogen ist hat es keine Kirschen unerlaubt gegessen. In seinem darauf folgenden nächtlichen Traum hat das Kind den Korb mit Kirschen vor sich gesehen und alle aufgegessen. Das Unbewusste hat im Traum zur Wunscherfüllung gedrängt, was Morgenthaler allerdings für jeden Traum annimmt. Zudem sieht er die Wunscherfüllung, die Kirschen verzehren zu wollen, bereits als eine unbewusste Ich-Leistung. Diese hat sich an der Erfahrung gebahnt, Kirschen gepflückt zu haben und den erzieherischen Einflüssen der Eltern gefolgt zu sein. Für Morgenthaler ist auch das interessant, doch ist er viel mehr an den Auswirkungen der unbewussten IchAnteile interessiert.

Dieses Kind hat den Traum nicht seiner Mutter, sondern dem Dienstmädchen erzählt. Erst von diesem hat die Mutter den Traum ihres Kindes erfahren das diesen Weg gewählt hat, wohlwissend, dass das Dienstmädchen den Traum weitererzählen würde. Da Morgenthaler zahlreiche Informationen über die Mutter, seine Analysandin, hatte, kam er zur Erkenntnis, dass das Kind Angst hatte, so manipuliert zu werden, wie es selbst im Traum die Kirschen manipuliert. «Es hat Angst, von der Mama gefressen zu werden, wie es selbst die Kirschen isst. Das will das Kind vermeiden, indem es den Traum dem Dienstmädchen erzählt ...» (Morgenthaler, 1990, S. 71). Das ist eine höchst spannende, nachvollziehbare Interpretation. Interessanterweise erfährt der Leser nicht, ob es sich bei dem «Kind» um einen Jungen oder ein Mädchen gehandelt hat, ein solches Wissen könnte nochmals ein ganz unterschiedliches unbewusstes Begehren der Mutter verraten. Mit diesem Traumbeispiel macht Morgenthaler eindrücklich deutlich, dass es bei der Traumdiagnostik nicht genügt, sich mit den Kenntnissen der Traumtheorie zu begnügen, sondern dass auf alle unbewussten Tendenzen zu achten ist. In ihrem Vorwort zu Morgenthalers Werk «Der Traum» weisen Paul Parin und Hans-Jürgen Heinrichs darauf hin, dass sich Morgenthaler ganz selbstverständlich immer auf das bestehende metapsychologische Gerüst gestützt hat. Im Vordergrund stand für ihn jedoch der emotionale Austausch, den er nicht durch Regeln und Gesetze beeinträchtigt wissen wollte (Morgenthaler, 1990, S. 9). Es ist also ratsam, nicht bei den Inhalten eines Kindertraums zu verharren, vor allem nicht bei den angeblichen Einschränkungen, sondern nach der Tendenz in der Dynamik eines Traums zu suchen: In welche Richtungen gehen seine Bedürfnisse (vgl. auch Binswanger, 2008, S. 59)?

\section{Ein Kindertraum unter verschiedenen Aspekten}

Wie sich die Suche nach der Tendenz und der Dynamik eines Traums ausgestalten kann, will ich am Beispiel eines psychisch gesund entwickelten Kindes aufzeigen. Dabei werde ich nicht nur den Traum untersuchen, sondern auch den Einfluss der Tochter-Mutter-Beziehung auf das Geträumte. Alle Kinderträume haben, wie in der analytischen Situation, einen Adressaten - in der Familie sind es zumeist Mutter oder Vater, seltener ein Geschwister.

Marlene, drei Jahre und zehn Monate alt, hat das Folgende geträumt. Nach dem Aufwachen hat sie den Traum ihrer Mutter erzählt: «Ich habe heute geträumt, dass ich bei der Oma war und fliegen konnte. Ich war bei der Oma und konnte durch das Haus fliegen.»

Auslöser (der sogenannte Tagesrest) für diesen Traum war, dass Marlene mit Bruder Leonard bei der Oma zu Besuch gewesen war. Dort haben sie vorgespielt, was sie kürzlich mit ihrem Kindergarten aufgeführt hatten. Leonard hatte im Chor mitgewirkt und Marlene hatte getanzt. Bei Oma und Opa hat Leonard das Lied vorgesungen («Alles ist so leicht beim Fliegen, leg Dich einfach auf den Wind») und Marlene ist mit den Armen schwingend, das Fliegen imitierend, durch den Raum «geschwebt». Beide waren über die anerkennenden Worte von Oma und Opa und deren Bewunderung ihrer Darbietungen erkennbar glücklich. Ein Tagesrest kann immer unbewusste Konflikte auslösen. Was war hier vermutlich geschehen?

Im Haus der Oma erobern die Kinder in kurzer Zeit alle Zimmer mit ihren Spielsachen und «fliegen» durch das ganze Haus, was sie zu Hause so unbegrenzt nicht dürfen. Das wünscht sich Marlene jedoch sehr.

Bereits wenn ein Kind das aufrechte Gehen erlernt, erlebt es das wahrscheinlich so, als könne es die Welt regelrecht beherrschen. Der Traum vom Fliegen entspricht einem bekannten Muster. Er ist ein Abbild von grandiosen Gefühlen und von dem Wissen, etwas Einzigartiges zu können. Auch in Marlenes Traum wird mit ihrem Fliegen nicht nur die Erinnerung an die Aufführungen wiederholt, sondern es werden die damals erfahrenen Hochgefühle wiederbelebt. Der Traum besitzt aber auch eine kommunikative Funktion - er ist eine Botschaft an die Eltern, insbesondere an die Mutter.

Als Marlene nach ihrem Besuch bei der Oma von den Eltern wieder abgeholt wurde, weinte sie heftig und äusserte wütend: «Ich will bei der Oma bleiben.» Im Traum hat sie sich diesen Wunsch erfüllt. Die Oma wird im Traum sogar zweimal erwähnt. Triumphierend kann sie der Mama mitteilen: «Ich bin, auch gegen Deinen Willen, bei der Oma geblieben.» Marlene ist auf dem Höhepunkt ihrer Autonomieentwicklung und betont darum ihre Selbständigkeit, ihre Unab- 
hängigkeit, besonders stark. Würden wir einen Fokus bilden, so hiesse der: «Ich mache, was ich will, denn ich bin gross und stark.»

Träume von kleinen Kindern sind also nicht nur simple Wunscherfüllungen, die wenig aussagen. Damit hat Freud lediglich die Inhalte betrachtet, nicht jedoch die Tendenzen und die kommunikativen Aspekte.

Ich fasse zusammen: Als Teilnehmer an diesen Szenen (ich bin der Grossvater) kannte ich die Tagesreste, teilweise aus eigener Wahrnehmung, teilweise aus den Erzählungen der Mutter.

Auch ist die Wunscherfüllung leicht zu erkennen, nämlich alle Zimmer zu besetzen und beherrschen zu wollen.

Eindrücklich wird deutlich, dass jeder Traum auch eine Selbstdarstellung ist. Marlenes Traum demonstriert Gefühle von Grandiosität, Überlegenheit und Herrschaft über die anderen. Diesen Aspekt nennen wir auch subjektstufig. Am wichtigsten scheint mir, dass der Traum eine kommunikative Funktion besitzt und hier eine Botschaft an die Eltern darstellt. In einer psychoanalytischen Therapie ist ebenfalls bereits eine Adressatin oder ein Adressat vorhanden - wie auch die Stunde, in welcher der Traum erzählt oder verschwiegen werden kann (Binswanger, 2008, S. 53)

Dieser Traum ist typisch für die Entwicklungsphase des noch nicht vierjährigen Mädchens. Noch geht es um Loslösung und um die Durchsetzung eigener Machtansprüche zwischen analer und phallischer Phase.

Ich habe zu Beginn keinen Traum aus einer Kinderpsychoanalyse, sondern den Traum eines noch kleinen Mädchens innerhalb seiner Familie gewählt. Damit habe ich die vielfältigen Botschaften von Träumen sehr kleiner Kinder zu verdeutlichen versucht - und dass Kinderträume darum auch in der psychoanalytischen Praxis immer in mehrfacher Weise verstanden und gedeutet werden können.

Im Traumbeispiel der kleinen Marlene war zu erkennen, dass sie ihren Traum keineswegs zufällig, sondern mit einer unbewussten, vielleicht sogar teilbewussten Absicht der Mutter erzählt hat.

\section{Ein Initialtraum}

Mit der folgenden Fallgeschichte aus der zweiten Sitzung einer Kinderpsychoanalyse, will ich darlegen, wie wichtig es im therapeutischen Setting ist, die unbewussten Absichten des Patienten, vor allem seine spezifischen Übertragungen zu erkennen (vgl. Hopf, 2007, S. 106f.). Ich will es nochmals betonen:Verweilen wir lediglich bei der Deutung der Trauminhalte, so bleiben die zentralen Tendenzen des Träumers verborgen.
Felix, 12 Jahre alt, kam wegen Schulversagens und depressiven Verstimmungen zu mir in analytische Psychotherapie. Rasch wurde deutlich, dass nicht Felix das Problem war, sondern seine Mutter; die ausserordentlich rigide, streng und kontrollierend war. Sie hatte sich von ihrem Mann getrennt, weil ihr dieser «zu weich und passiv» gewesen wäre und wollte jetzt ihren Sohn zu «Leistungsbereitschaft und Erfolg im Leben» erziehen. Sie suchte therapeutische Hilfe, weil sich der Sohn «ähnlich schlaff wie sein Vater» zeigte. Schon im ersten Gespräch hatte sie sich eindringend, kontrollierend und bestimmend gezeigt, sodass ich mir ernsthaft überlegte, ob eine Therapie von Felix mit dieser Mutter überhaupt möglich wäre. Obwohl er vom Alter her bereits Jugendlicher war, müsste unbedingt mit seinen Eltern gearbeitet werden, weil die Mutter ansonsten rasch für einen Abbruch sorgen würde.

Bereits in der zweiten Stunde berichtete Felix von einem Traum, der ihn «sehr aufgeregt» hätte.

Ich habe geträumt, ein Mann operiert mir die Warzen an meinem Bein weg. Dabei hat er höhnisch gelacht. Er sah aus wie der Joker aus Batman. Dann hat er mir mein Bein wegoperiert. Ich habe Angst gekriegt und bin aufgewacht. Danach habe ich nach meinem Bein gegriffen und war froh, dass es noch da war.

Zum Traum hatte Felix die folgenden Assoziationen: Seine Mutter musste zum Hautarzt, und Felix musste sie dorthin begleiten. Als sie dort waren, meinte sie, wenn er schon mal mit hier wäre, könnte der Arzt auch gleich mal seine Warzen anschauen. Dieser schaute sie dann allerdings nicht nur an, sondern operierte sie gleich weg, ohne ihn zu fragen. Das sei sehr schmerzhaft gewesen und er habe noch abends voller Schmerzen im Bett gelegen. In der darauf folgenden Nacht hätte er dann diesen quälenden Traum gehabt.

Offensichtlich ist die Angst von Felix seiner Mutter gegenüber so gross, dass er alle Eigenschaften, die er bei ihr hasst, dem Arzt zugeschrieben hat. Dieser ist rücksichtslos, achtet ihn und seine eigenen Wünsche nicht, und er kastriert ihn. Ich habe Felix' Traumbild auch als Übertragungsangebot erlebt: Seine Mutter hatte ihn bei mir, wie beim Hautarzt abgeliefert; ich sollte ihn behandeln, ohne dass ich mit ihm ausreichend darüber gesprochen hätte, ob er das überhaup wollte. Felix hatte selbstverständlich einer Behandlung bei mir - vordergründig zugestimmt. Er fühlte sich jedoch hilflos und ausgeliefert, er fürchtete, von mir so kastriert und «zurecht- und glattgehobelt» zu werden, wie das seine Mutter 
wünschte. Nach dem Aufwachen stellte er allerdings fest, dass er sein Bein (seinen Phallus?) noch hatte - diesen Teil habe ich als ersten, leisen Triumph empfunden. Auch wenn der Initialtraum nicht gedeutet werden soll, habe ich die bewussten Anteile des Traumes aufgegriffen und zu Felix gemeint, dass es wohl oft Situationen gäbe, in denen er sich hilflos und ausgeliefert erlebte. Aber der Traum hätte ihn offensichtlich auch aufgeregt; ich ginge darum davon aus, dass er das so nicht belassen wollte. Daraufhin haben wir mit der Therapie begonnen, die zu einem guten Ergebnis geführt hat. Dieser Initialtraum hat zur Etablierung eines stabilen Behandlungsbündnisses erheblich beigetragen.

Der erste Traumbericht in einer Behandlung, auch wenn er erst in späteren Stunden erfolgt, wird Initialtraum genannt. Er folgt in der Regel auf die Etablierung einer einigermassen stabilen Beziehung und einer positiven Übertragung. Der Initialtraum zeigt die gesamte Psychodynamik auf, die für den Analytiker allerdings nicht immer ganz zu verstehen ist, und er stellt einen Versuch des Patienten dar, zu einer für ihn spezifischen, neuen Form von Kommunikation und Konfliktlösung zu gelangen. Der Initialtraum kann bis zur Beendigung der Analyse nutzbar gemacht werden, da er Aspekte von Gegenwart, Vergangenheit und Zukunft in sich vereinigt und aufzeigt, was erst die weitere Behandlung bestätigen oder erklären kann er enthält letztendlich das unbewusste Therapieprogramm (vgl. Becker, 1972). Der Initialtraum kann in seiner gesamten Vielschichtigkeit wohl erst am Ende einer Behandlung ganz verstanden werden. Diese Komplexität des Initialtraums macht Deutungen allerdings unmöglich (vor allem riskant!), weil das Ich des Patienten zu jenem Zeitpunkt in der Regel noch nicht imstande ist, ohne vermehrte Abwehr auf sie zu reagieren. Altman betont, dass durch vorschnelle Deutungen lediglich Angst und Abwehr verstärkt würden: «Vorsicht bei der Entwicklung des Behandlungsbündnisses soll unsere vornehmste Überlegung während der gesamten Analyse, besonders aber zu ihrem Beginn, sein» (1992, S. 55).

Wir betrachten die Traumerzählung mittlerweile als spezielle Form einer Erzählung, als ein sogenanntes Narrativ. Nach Boothe ist der Traum geradezu ein Prototyp für die in Eigenregie erfolgte Verwandlung von Erinnerung. Mit der Traumerzählung wird der vergangene Traum aktualisiert und neu erlebt. Die sprachliche Form der Traummitteilung lädt den Hörer zur Klärung und Aufklärung, zur Enträtselung ein, was die Alltagserzählung gerade nicht tut (Boothe, 2011, S. 69)

Wie bereits erwähnt, sind wir mittlerweile von verschiedenen Funktionen, auch des Kindertraums, überzeugt. Neben der Wunscherfüllung besitzen Träume eine kompensatorische sowie eine prospektive Funktion. Sie dienen der
Selbstdarstellung, haben eine kommunikative Funktion und eine adaptive: Träume dienen der Wiederherstellung des seelischen Gleichgewichts.

Rauchfleisch (1984) hat in einem Buchbeitrag den Traum einer Erwachsenen unter einigen Deutungsaspekten betrachtet. Dabei wollte er keiner der verschiedenen Zugangsmöglichkeiten Vorrang gewähren; vielmehr sollten die verschiedenen Aspekte einander ergänzen. Je nach Persönlichkeit des Patienten und nach Stand der Analyse kann seiner Meinung nach im Verlauf einer Behandlung einmal die eine, ein anderes Mal eine andere Interpretationsebene gewählt werden. Ich habe Rauchfleischs Kriterien teilweise übernommen, für Kinder und Jugendliche ergänzt und modifiziert. Ich will mit dem vorliegenden Interpretationsmuster verdeutlichen, dass, obwohl Kinderträume in der Regel kürzer sind als Träume Erwachsener, dieselbe Vielfalt erkennbar wird und auch aufgegriffen werden kann.

Im Folgenden stelle ich einige wichtige Kriterien dar, die sich problemlos auf Träume von Kindern übertragen lassen. Jeder Traum eignet sich dazu, mit dem dargestellten Interpretationsmuster untersucht zu werden. Eine ausführliche Darstellung erfolgt in meinem Buch Träume von Kindern und Jugendlichen (Hopf, 2007, S. 41 f.).

Meine ersten Fragen beziehen sich auf die Stimmung des Patienten beim Träumen und darauf, an welche Tagesreste er sich erinnert. Unter dem Tagesrest verstehen wir jene oft - vermeintlich - banalen Elemente aus dem Vortag, die der Traum als Anknüpfungspunkte verwendet. Dies kann das Bruchstück einer Wahrnehmung sein, eine Stimmung, eine Filmsequenz usw., die in eine Bildsequenz eingebaut werden. Ich nehme die Stimmungen, Emotionen und Affekte in mich auf und lasse sie auf mich wirken. Meine Gegenübertragungsaffekte lassen mich erste Ursachen für die Traumentstehung erkennen.

Als zweites betrachte ich die psychodynamische Seite: Welche Wünsche, welche Ängste finden ihren Ausdruck? Ich versuche dabei, Angststufen zu erkennen: namenlose Ängste, Todes- und Trennungsängste, Ängste vor Liebesverlust, Scham und Schuld ...

Da Kinderträume kurz, klar und kohärent sind, lässt sich leicht ein Fokus, ein zentraler Konflikt herausarbeiten.

Als nächstes schliesse ich vom Traum auf die Psychogenese der Persönlichkeit derTräumerin, des Träumers. Ich suche Hinweise auf die Entwicklungsbedingungen.

Unter Deutung auf der Objektstufe werden die im Traum auftretenden Personen als die realen Objekte oder Beziehungspersonen des Patienten aufgefasst. Der Traum liefert Hinweise auf die Qualität der Interaktionen mit den 
Beziehungspersonen (samt ihren gefühlsmässigen Komponenten), aber auch mit den Gegenständen und vorhandenen situativen Faktoren.

Bei einer Deutung auf der Subjektstufe wird der Traum als innere Bühne verstanden. Alle im manifesten Inhalt erscheinenden Personen oder Gegenstände stellen Repräsentanten verschiedener Seiten des Träumers dar. Alle Traumstücke, also auch handelnde Personen, werden auf den Träumer bezogen.

Wichtig ist es auch, Symbole zu erkennen. Gemäss der Freud'schen Auffassung vom individuell gestalteten Symbol gelingt es dem Träumer, über die Verwendung eines Symbols einen Kompromiss zwischen dem unbewussten Impuls und der Abwehr herzustellen. Da Symbole einen Versuch darstellen, unbewusste Motive zu verkleiden, sollten sie natürlich nicht gedeutet werden.

Über Träume als Ausdruck des Übertragungsgeschehens wurde bereits berichtet. Jeder Traum kann über die derzeitige Qualität der Übertragung des Patienten, d.h. seiner Gefühle dem Therapeuten gegenüber, etwas aussagen. Gemäss Ermann (2005, S. 70) wird die hinter einem Traumbericht stehende Dynamik der Traumerinnerung zum Schlüssel des Verständnisses der aktuellen Behandlungssituation. Letztendlich können wir die Bedeutung eines Traumes nur verstehen, wenn wir seine Verflechtungen im Beziehungs- und Behandlungsgeschehen analysieren.

\section{Ein Kindertraum in einer psychoanalytischen Behandlung}

Einige der genannten Kriterien möchte ich im Folgenden bei einem Traum anwenden, der mir innerhalb einer Supervision erzählt wurde. Bereits während der ersten probatorischen Sitzungen hat Jacqueline ihrer Therapeutin den folgenden Traum berichtet:

Grosse Würmer, grünbraun, sind unter der Haut, an Armen und Beinen, winden sich nach oben.

Es ist ein stark verdichteter Kindertraum, der unendlich viele Konflikte in sich birgt. Die Patientin, neuneinhalb Jahre alt, lebt seit einem Jahr im Heim. Die Vaterschaft ist unklar. Die Mutter, heute 35 Jahre alt, wurde von ihrem leiblichen Vater sexuell missbraucht, war massiv heroinabhängig und trinkt nach wie vor viel Alkohol. Sie ist Bäckereifachverkäuferin und arbeitet aushilfsweise in einem Supermarkt. Sie war zweimal verheiratet, es gab viele Beziehungen zu Männern, die sich möglicherweise auch gegenüber der Tochter übergriffig gezeigt haben. Eine Schwester der Patientin leidet an einer schweren Borderline-Störung Diese Schwester drohte, die Mutter umzubringen, war wegen Falschaussage im Gefängnis, ist jetzt wegen akuter Selbstgefährdung in einer geschlossenen psy- chiatrischen Klinik. Jacqueline erzählt, dass diese Schwester häufiger versucht habe, sie in der Elbe zu ertränken, sie auch aufs Fensterbrett gestellt habe, mit der Aufforderung, sie solle runterspringen.

Mutter und Tochter seien viel in Kneipen gewesen. Jacqueline habe immer im Bett ihrer Mutter geschlafen, wurde dort wahrscheinlich Zeuge von sexuellen Handlungen ihrer Mutter mit vielen Männern und erfuhr dabei vermutlich auch Übergriffe. Jacqueline verwahrloste, war viel allein unterwegs, nahm Hunde aus Gärten mit, wurde nachts von der Polizei aufgegriffen. Sie habe getreten, geschlagen, Wutausbrüche gehabt. Wegen ihres impulsiven Verhaltens wurde die Patientin zeitweise aus der Schule ausgeschlossen. Aufgrund dieser Vorkommnisse wurde das Mädchen schliesslich aus der Familie herausgenommen.

Wie bereits erwähnt ist die Wahrnehmung der eigenen Gestimmtheit mit allen Gegenübertragungsreaktionen ein wichtiges Deutungskriterium, das bei jedem Traum Anwendung finden kann. Die Erzählung des Traums löste heftige Affekte bei der Therapeutin und innerhalb der Supervision aus. Sie schwankten zwischen Erschrecken, Entsetzen, Grausen, Angst und - vor allem - Ekel. Ekel ist bekanntlich einer der heftigsten Affekte, die einen Menschen erfassen können. Er ist ein Alarm- und Ausnahmezustand und betrifft alle körperlichen Reaktionen. Vom Soziologen Menninghaus stammt die Aussage, dass das elementare Muster des Ekels die Erfahrung einer ungewollten Nähe ist.

Gleichzeitig wurden Gefühle von Hilflosigkeit und von Ausgeliefertsein wahrgenommen. Die Farben im Traum erinnerten an Auswurf und an Exkremente. Das erste Deutungskriterium «Gestimmtheit» erbrachte bereits die meisten Hinweise über die Hintergründe dieses Traumes. Wir können davon ausgehen, dass die jetzigen Gegenübertragungswahrnehmungen jenes Erschrecken und Grausen und jenen Ekel wiedergeben, die Jacqueline wahrscheinlich während der Nächte im Bett der Mutter erlebt hat. Diese entsetzlichen Affekte haben sich tief in ihre Erinnerungen eingegraben und erzeugen vermutlich immer wieder traumatische Träume. Im Mittelpunkt stehen, wie bei traumatischen Träumen üblich, Schutzverlust, Verlassenheit, Ekel, Wut, Angst ...

Als typische Angst ist eine panikartige Angst vor Nähe zu erkennen, der Wunsch im Traum ist, dass ihr unbedingt geholfen werden möge. Das ist auch gleichzeitig das Übertragungsangebot. Die Therapeutin verspürte ein intensives Bedürfnis, ihrer Patientin beizustehen und zu helfen.

Betrachten wir die Inhalte hinsichtlich der Objektstufe: Es existieren keine lebenden Objekte, die Träumerin ist allein den schrecklichen «grossen Würmern» ausgeliefert - sind es womöglich Penisse? 
Subjektstufig existieren wenig stabile Repräsentanzen. DieWürmer winden sich nach oben, drohen, auch das Bewusstsein zu zerstören. Autoaggression wird erkennbar.

Symbolik: DerWurm ist im Laufe der Geschichte zu einer Metapher für heimtückische Zerstörungswut geworden. In ihn wird auch Niedrigkeit und kriecherisches Verhalten projiziert. Symbolisch wird derWurm mit Tod und Verwesung sowie dem Zerfall von Leichen verbunden. Andererseits existieren Vorstellungen, dass über die Zerstörung des Toten erst ein Neubeginn möglich wird. Das ist vielleicht ansatzweise eine Ressource des Mädchens. Dennoch handelt es sich um einen «nieder strukturierten» Traum; er demonstriert uns die wenig verkleidete Darstellung des bedrohten «Ich-Zustandes» der kleinen Träumerin. Dieser Traum wirkt anders als die meisten alltäglichen Träume, die während einer Therapie berichtet werden. In Träumen werden unsere geheimen Wünsche, unsere Absichten, auch Triebhaftes und «niedere» Beweggründe verkleidet und unkenntlich gemacht. Dazu verfügt die Traumarbeit über bestimmte Mechanismen der Entstellung. Einer der wichtigsten ist die Symbolisierung. Auf diese Weise kann Eindeutiges vieldeutig werden. Aus gutem Grund sollen unsere unbewussten Wünsche bedeckt gehalten werden, denn unsere Träume sollten uns nicht allzu sehr beunruhigen, sondern vor allem den Schlaf hüten. Jacquelines Traum setzt jedoch grausame Wirklichkeiten unverhüllt in Szene. Sie werden nicht mehr hinter Symbolisierungen verborgen. Solche Träume sind nach Traumatisierungen gang und gäbe, weil die symbolisierenden Fähigkeiten durch das Trauma oft zerstört, zumindest eingeschränkt worden sind Es können jetzt Träume auftreten, welche die innere Gefährdung unmittelbar darstellen. Der Traum des kleinen Mädchens schildert die real erfahrenen furchtbaren Ereignisse und das dazugehörige Empfinden: Er zeichnet den Schrecken nach, den Jacqueline erlebt hat und verleiht den damaligen Gefühlen eindringliche Bilder.

Erst wenn angstmachende Themen und Traumata im Rahmen zwischenmenschlicher Beziehungen wieder belebt werden, sind sie einem therapeutischen Einfluss zugänglich. Nicht das Träumen allein ist entscheidend, auch nicht eine noch so weise Interpretation, sondern die Reinszenierung des Traumas und seine «Bebilderung» in einer haltenden und «containenden» Beziehung. Jacquelines Traum war also eine bildliche Darstellung ihrer nicht verinnerlichten affektiven Erfahrungen. «Containend» verstehe ich so, dass der Therapeut die Gefühle aufnimmt und sie schon dadurch verändert.

\section{Abschliessende Bemerkungen}

Der Kindertraum ist eine ebenso wunderbare via regia zum Unbewussten wie derTraum eines Erwachsenen. Mit einerTraumerzählung können wir die unbewussten Konflikte des Träumers rasch erfassen und Wege zur Bewältigung erfahren. Mittlerweile sind wir auch nicht mehr auf die Assoziationen der Träumer angewiesen, weil wir vielfältige Zugangswege kennen, um den manifesten Trauminhalt zu dechiffrieren; der manifeste Trauminhalt ist nicht mehr die nutzlose Schale, wie einst von Freud gewertet, sondern letztendlich der wertvolle Kern.

Binswanger hat Morgenthalers Erkenntnisse zusammengefasst, warum verbale Assoziationen nicht unbedingt erforderlich seien:

Alles, was in derselben Analysestunde geschieht und erzählt wird, betrachtet er als Assoziationen zum erzählten Traum, unabhängig davon, ob es vor oder nach dem Traum erzählt wurde, ob es inhaltlich dazu gehört oder nicht, ob es bedeutungsvoll oder als Nebensächlichkeit daherkommt. Nicht nur, was der Analysand erzählt, sondern auch alles, was er szenisch, mimisch oder gestisch darstellt, was er mit dem Analytiker anstellt und inszeniert, ist als Assoziation zum Traum zu betrachten. (Binswanger, 1990, S. 182 f.)

Wir brauchen auch keine Angst zu haben, zu intrusiv ins Unbewusste des Kindes einzudringen. Gemäss Winnicott kann ein Traum immer in der Therapie genutzt werden, da die «Tatsache, dass er geträumt, erinnert und berichtet wird, anzeigt, dass sich das Traummaterial und die dazugehörigen Ängste und Erregungen im Bereich der Kapazität des Kindes befinden» (1973, S. 99). Und hinsichtlich der Deutungsarbeit meinte Winnicott: «Interpretiert wird nur wenig. Eine Interpretation ist nicht in sich therapeutisch, sie fördert lediglich das, was therapeutisch ist, nämlich das erneute Durchleben angsteinflössender Erfahrungen. Mit Hilfe der Ich-Stärkung durch den Therapeuten, kann das Kind diese SchlüsselErlebnisse zum ersten Mal in die Gesamtpersönlichkeit assimilieren» (a.a.O., S. 180). Somit ist eine solche Arbeit mit Träumen vollkommene Kommunikation und schon das kleinste Kind begreift mit seinem Analytiker unbewusste Konflikte.

Nichts erhellt eine aktuelle Konfliktsituation, vorhandene Ängste, die Ichstärke und Symbolisierungsfähigkeit besser als ein Traum. Aber wie gelingt es, Kinder auch zum Erzählen ihrer Träume zu motivieren? Aus Traumseminaren ist bekannt, dass das manchmal als Problem erlebt wird. Oft besteht aus einer falsch verstandenen Abstinenz-Haltung heraus eine Scheu, Kinder direkt nach ihren 
Träumen zu fragen. Nach meiner Erfahrung erzählen Kinder ihre Träume gerne und machen sie damit auch gerne dem Analytiker zum Geschenk. Natürlich sind Traumerzählungen immer in das gesamte Beziehungsgeschehen mit Übertragungen und Widerständen eingebunden. Unsere positive Haltung Träumen gegenüber ist entscheidend - spüren die Kinder diese, werden sie uns viele erzählen.

\section{Literatur}

Ablon, S.L. und Mack, J.F. (1980). Children's Dreams Reconsidered. The Psychoanalytic Study of the Child 35, 179-217.

Altman, L. L. (1992). Praxis der Traumdeutung. Frankfurt a. M.: Suhrkamp Taschenbuch.

Becker, A. (1972). Zum Initialtraum. Psyche-Zeitschrift für Psychoanalyse und ihre Anwendungen 26, 689-706.

Binswanger, R. (2008). Freud - Morgenthaler. Verknüpfung der Traumtheorien. Werkblatt 60, 25(1), 47-62.

Binswanger, R. (1990). Zur Traumtechnik Fritz Morgenthalers im Traumseminar und in der psychoanalytischen Praxis. In Morgenthaler, F. (1990), Der Traum. Fragmente zur Theorie und Technik der Traumdeutung. Frankfurt, New York: Campus Verlag.

Boothe, B. (2011): Das Narrativ. Biografisches Erzählen im psychotherapeutischen Prozess. Stuttgart: Verlag Schattauer.

Burchartz, A., Hopf, H. \& Lutz, C. (2016). Psychodynamische Therapien mit Kindern, Jugendlichen und jungen Erwachsenen. Geschichte, Theorie, Praxis. Stuttgart: Verlag W. Kohlhammer.

Despert, J. L. (1949). Dreams in Children Preschool Age. Psychoanalytic Study of the Child. 3-4, New York: International Universities Press, 141-180.

Erikson, E.H. (1955). Das Traummuster der Psychoanalyse. In Graevenitz, J.v. (Hg.), Bedeutung und Deutung des Traumes in der Psychotherapie. Wissenschaftliche Buchgesellschaft: Darmstadt.

Ermann, M. (2005). Träume und Träumen. Hundert Jahre «Traumdeutung». Stuttgart: Verlag W. Kohlhammer.

Freud, A. (1978). Einleitungen zu Band 1 und Band 2 der Sigmund-FreudWerkausgabe in zwei Bänden. Die Schriften der Anna Freud, VIII, München: Kindler Verlag, 1980.

Freud, A. (1965). Wege und Irrwege in der Kinderentwicklung. Die Schriften der Anna Freud, VIII. München: Kindler Verlag, 1980
Freud, S. (1916/1917). Vorlesungen zur Einführung in die Psychoanalyse Studienausgabe Bd. I. Frankfurt a. M: S. Fischer Verlag, 1969.

Freud, S. (1900a). Die Traumdeutung. Studienausgabe Bd. II. Frankfurt a. M. S. Fischer Verlag, 1972.

Hopf, H. (2015). Kinderträume verstehen. Frankfurt a. M.: Mabuse-Verlag. 2. überarbeitete und aktualisierte Auflage.

Hopf, H. (2007). Träume von Kindern und Jugendlichen. Diagnostik und Psychotherapie. Stuttgart: Verlag W. Kohlhammer.

Hopf, H. (1985). Träume in der Behandlung von Kindern und Jugendlichen mit prä-ödipalen Störungen. In Hopf, H. (2005) (Hrsg.), Traum, Aggression und heilende Beziehung. (S. 43-54). Frankfurt a. M.: Edition Déjà-vu Verlagsabteilung der Sigmund-Freud-Buchhandlung.

Menninghaus, W. (1999). Ekel. Theorie und Geschichte einer starken Empfindung. Frankfurt a. M.: Suhrkamp Verlag.

Morgenthaler, F. (1990). Traumdiagnostik. Zur Bedeutung der formalen und strukturellen Gesichtspunkte. In Morgenthaler, F. (1990), Der Traum. Fragmente zur Theorie und Technik der Traumdeutung. Frankfurt, New York: Campus Verlag.

Parin, P. \& Heinrichs, H.-J. (1990). Vorbemerkungen. In Morgenthaler, F. (1990), Der Traum. Fragmente zur Theorie und Technik der Traumdeutung. Frankfurt, New York: Campus Verlag.

Rauchfleisch, U. (1984). Verschiedene Deutungsaspekte des Traumes. In WagnerSimon, T. \& Benedetti, G. (Hrsg.), Traum und Träumen. Göttingen: Verlag Vandenhoeck \& Ruprecht.

Winnicott, D.W. (1973). Die therapeutische Arbeit mit Kindern. Mit einer Einführung von M. Masud R. Khan. München: Kindler Studienausgabe.

Zierl, W. (1973). Die Rolle des Traumes in der Psychotherapie des Jugendlichen. In Biermann, G. (Hrsg.), Handbuch der Kinderpsychotherapie. Bd. 1. München Ernst Reinhardt Verlag.

\section{Angaben zum Autor}

Hans Heinz Hopf, Dr. rer. biol. hum., ist analytischer Kinder- und Jugendlichen-Psychotherapeut, bis 1995 in eigener Praxis, danach Therapeutischer Leiter im Therapiezentrum «Osterhof», Baiersbronn. Dozent und Kontrollanalytiker an den Psychoanalytischen Instituten Stuttgart und Würzburg. Seit 2003 wieder in eigener Praxis tätig. Psychotherapie-Gutachter für analytische und tiefenpsycholo- 
gisch fundierte Psychotherapie bei Kindern und Jugendlichen. Diotima-Ehrenpreis der Deutschen Psychotherapeutenschaft im Jahr 2013. 